\title{
AUTOEVALUACIÓN INSTITUCIONAL COMO ESTRATEGIA DE MEJORA PARA UNA ESCUELA BASADA EN DERECHOS DE LOS NIÑOS, NIÑAS Y ADOLESCENTES
}

\author{
Olga Graciela Córdova Lovera
}

\begin{abstract}
RESUMEN
Se presenta el Sistema de Autoevaluación, implementado en el Modelo de Escuelas Felices e Integrales (EFI), un espacio educativo centrado en los derechos de los niños, niñas y adolescentes sustentado en base a tres enfoques: el de Derechos, el de Equidad y el Participativo. También nos muestra cuál es el procedimiento para realizar la autoevaluación de manera participativa y contextualizada, y se concluirá sustentando el por qué el Sistema de Autoevaluación de las escuelas EFI es una adecuada estrategia para la mejora del servicio escolar, y cómo este sistema de autoevaluación, que es único y adecuado para el contexto del distrito de Ventanilla, puede ser replicado en otras escuelas que tengan también como centro al estudiante.
\end{abstract}

\section{PALABRAS CLAVE}

Autoevaluación institucional, indicadores, mejora continua, derechos, equidad, participación.

\section{ABSTRACT}

The Self-Evaluation System is presented, implementing in the Model of Happy and Integral Schools (EFI) an educational area focused on the rights of children and adolescents based on three approaches: Rights, Equity and Participatory. It also shows us the procedure for conducting the self-assessment in a participatory and contextualized way, and it will be concluded that the EFI school Self-Assessment System is an adequate strategy for the improvement of the school service, and how this evaluation system, which is unique and suitable for the context of Ventanilla district, can be replicated in other schools that also have as a student center.

\section{KEYWORDS}

Institutional self-evaluation, indicators, continuous improvement, rights, equity, participation.

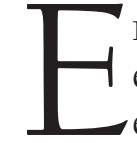
ncontrar espacios de aprendizaje donde los estudiantes sean felices y se les brinde una enseñanza de calidad a través de una convivencia alegre en donde se evidencie el respeto a los derechos del estudiante, es un verdadero reto. ¿De qué manera las instituciones educativas pueden reformular sus prácticas y brindar una educación de calidad centrada en los derechos de los niños, niñas y adolescentes?

Actualmente se presentan muchas alternativas; sin embargo, pensamos que los procesos de Autoevaluación Institucional se convierten en la principal estrategia para que los actores educativos puedan realizar una reflexión crítica sobre su propósito y su actuar, en relación a los objetivos institucionales que plantea y la calidad de educación que brinda.

De acuerdo con el Reglamento de la Ley № 28740, Ley del Sistema Nacional de Evaluación, Acreditación y Certificación de la Calidad Educativa, SINEACE, la autoevaluación institucional es el proceso de evaluación orientado a la mejora de la calidad y llevado a cabo por los propios actores; es decir, estudiantes, egresados, docentes, administrativos, autoridades, padres de familia y grupos de interés. Esta ley norma los procesos de evaluación, acreditación y certificación de la calidad educativa, define la participación del Estado en ellos y regula el ámbito, la organización y el funcionamiento de este sistema de evaluación; y referente a lo estipulado en los artículos $14^{\circ}$ y $16^{\circ}$, el Estado garantiza el funcionamiento de un Sistema Nacional de Evaluación, Acreditación y Certificación de la Calidad Educativa, que abarca todo el territorio nacional y responde con flexibilidad a las características y especificidades de cada región. 
La autoevaluación, entonces "no es autorreferencial, está orientada por el modelo establecido que facilita la identificación de fortalezas y debilidades en la gestión de la Institución Educativa (IE), para tomar decisiones de mejora" (IPEBA 2012).

Es en este marco legal se implementa el sistema de autoevaluación y mejora en las 14 Instituciones Educativas pertenecientes al modelo Escuelas Felices e Integrales (EFI). Las escuelas EFI son el Modelo de Escuela que busca mejorar la calidad educativa y el cumplimiento de los derechos de los niños, niñas y adolescentes, y es impulsado por el gobierno localde Ventanilla/Callao.

Estas 14 escuelas pertenecen a la Red Norte de Villa Los Reyes de Ventanilla, y la experiencia de autoevaluación nace de una necesidad de mejorar los servicios que brindan las escuelas de manera permanente y velar por el cumplimiento de los derechos de sus estudiantes. Es por ello que se busca que la autoevaluación sea parte de la cultura institucional en cada una de las escuelas y permita ver a todos los actores, sus avances en relación a lo propuesto en su proyecto educativo institucional, para el logro de las políticas locales, regionales y nacionales (Delgado 2013).

En las Escuelas Felices e Integrales, se propicia que los estudiantes tengan aprendizajes significativos, por ello el proceso de autoevaluación se centra en los siguientes enfoques:

\section{Enfoquedederechos}

En el marco de un proceso de Autoevaluación el enfoque de derechos es considerado desde dos planos de análisis: por un lado, en relación con los alumnos y, por otro, en relación con los docentes, directivos y familiares.

- Respecto de los derechos de los niños, se reconoce:

El derecho a la inclusión educativa en condiciones de equidad, tanto en el acceso como en la calidad de la educación que reciben. Esto incide en las posibilidades de desarrollo futuro personal y colectivo de los niños.

La escuela como entorno protector de los derechos de los niños (mediante acciones de prevención, detección y atención de vulneraciones de esos derechos).

- Respecto de los docentes y familiares, se considera:

El derecho a la participación del plantel docente: identificación de problemas, priorización y propuestas para la implementación de acciones de mejora.

El derecho de los familiares o adultos responsables a participar, opinar, tener acceso a información sobre el proceso de aprendizaje de sus hijos y proponer acciones en su beneficio.

\section{Enfoque de equidad}

El Enfoque de Equidad alude a la igualdad de oportunidades y logros de igual calidad para todos los estudiantes al margen del nivel socioeconómico, lugar de procedencia, lengua de los estudiantes, género, discapacidad o el tipo de IE a la que asisten. Al respecto, el Proyecto Educativo Nacional, oficializado como política de Estado en enero de 2007, enfatiza "la necesidad de lograr que todos los niños, niñas y adolescentes peruanos tengan las mismas oportunidades educativas y alcancen resultados de igual calidad al final de la Educación Básica".

\section{Enfoqueparticipativo}

Es una forma de práctica investigativa, en la cual grupos de personas organizan sus actividades con el objetivo de mejorar sus condiciones de vida y aprender de su propia experiencia, atendiendo a valores y fines compartidos. Su modelo constituye una espiral permanente de reflexión y acción, fundamentado en la unidad entre la práctica y el proceso investigativo, que se desarrolla a partir de las decisiones del grupo, el compromiso y el avance progresivo.

Desde este punto de vista, entendemos un verdadero desarrollo como un proceso "sin fin" y suman a las necesidades materiales básicas, sus necesidades intangibles y psicológicas. Asimismo, partimos de un concepto de desarrollo como la capacidad de producir simultáneamente y en armonía, el crecimiento económico y el desarrollo social, en base a cada cultura local. 
La participación activa en diferentes espacios de vida favorece un análisis del entorno más inmediato, en este sentido, el enfoque participativo se convierte en la herramienta idónea para llevar a cabo el proceso autoevaluativo, ya que propicia espacios de reflexión colectiva en torno a la gestión educativa, sus resultados alcanzados, que puede llevarlos a una nueva planificación y a cambios sucesivos, en donde los actores asumen compromisos desde la acción activa y directa en las acciones y procesos institucionales, pedagógicos y administrativos, ofreciendo propuestas de mejora y dinamizando cambios que evidencien calidad.

Es importante resaltar que este proceso de autoevaluación de las Escuelas Felices e Integrales es holístico, participativo e integral; por lo tanto, las escuelas se autoevaluarán en relación a la mejora de los aprendizajes, pero teniendo en cuenta variables como salud, nutrición, buen trato y adecuada infraestructura que conviertan a las escuelas en espacios acogedores y agradables para generar mejores aprendizajes en los estudiantes.

El Gráfico 1 permite ver la articulación entre los componentes del modelo y el proceso de autoevaluación de las escuelas EFI.

\section{Gráfico 1}

Articulación del Sistema de Autoevaluación y los Componentes del Modelo EFI

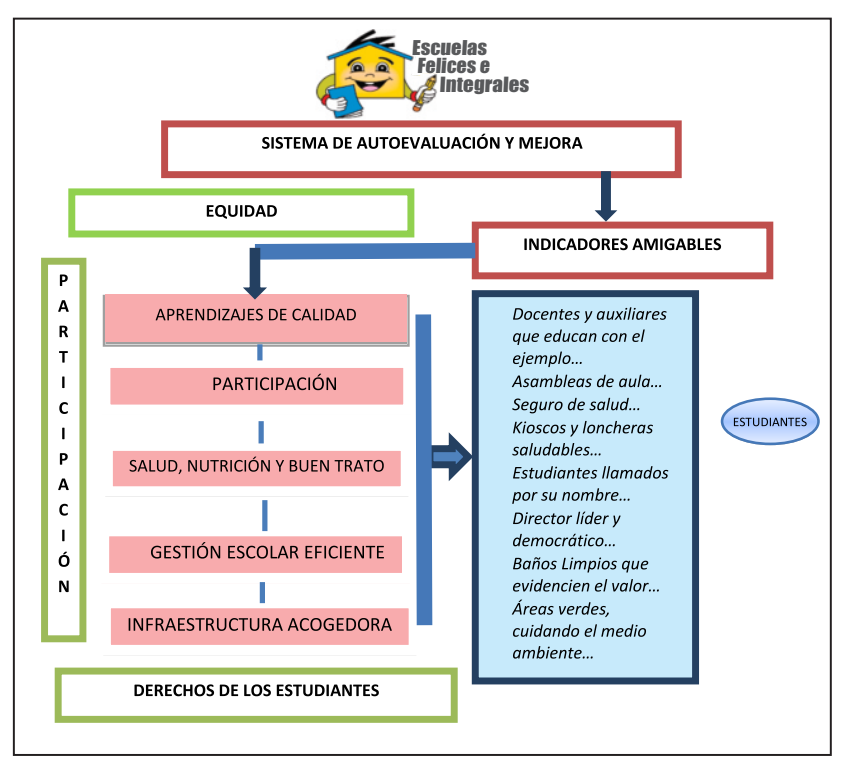

La autoevaluación institucional, como una de las tareas fundamentales de la gestión directiva, se encuentra a cargo del Consejo Educativo Institucional (CONEI). Ellos son los encargados de dirigir el proceso para coordinar recursos y equipos, asignación de roles y tareas y velar por la calidad del resultado.

A continuación se detallan los pasos de la Autoevaluación Institucional que se ha construido en el modelo EFI con la finalidad de orientar y facilitar esta tarea. Estos son:

\section{Pasol-Autoevaluación inicial}

No se puede llevar a cabo procesos de evaluación sin planificar la acción evaluativa. Esta debe ser sistemática, organizada e intencionada. Para ello, se realizará el plan de autoevaluación de manera participativa y democrática, y además, efectivizar el trabajo sabiendo qué, cómo, quiénes y cuándo desarrollaremos el proceso de autoevaluación.

Es en esta etapa donde se realizan reuniones para conocer a profundidad el proceso, los componentes del modelo y los indicadores amigables, teniendo como objetivo el lograr que toda la comunidad educativa conozca sobre el proceso y su importancia en el desarrollo de la escuela.

Esta etapa comprende también la sensibilización y difusión del proceso de autoevaluación y mejora, en donde se busca crear las condiciones favorables para que la comunidad educativa participe motivada y se comprometa con el proceso.

Como parte de la sensibilización y difusión del proceso se pueden realizar periódicos murales, artículos, eslogan, que propicien el conocimiento del proceso en toda la comunidad educativa.

Paso 2-Selección de indicadores amigables y aplicación de instrumentos

La selección de los indicadores amigables está centrada en la orientación a la promoción, respeto y defensa de los derechos de los estudiantes, mediante la participación y compromiso de la 
comunidad educativa. En tal sentido, la educación es un medio que permite el cumplimiento efectivo de otros derechos, como la igualdad de oportunidades para aprender, sin que estas igualdades sean afectadas por razones de género, cultura, discapacidad o de cualquier otra índole. Es en este paso donde se evidencia la capacidad de autorreflexión de los actores educativos.

Los indicadores amigables están organizados por los componentes del modelo, de tal manera que nos permite evaluarnos institucionalmente. En el Cuadro 1 se presenta un modelo de indicadores priorizados por una escuela de la red, pero teniendo en cuenta la característica de singularidad del proceso, cada escuela está en libertad de seleccionar o proponer otro indicador en relación con el componente del modelo a evaluar.

\section{Cuadro 1}

Indicadores priorizados de la IEI 86 "Corazón de María"

\begin{tabular}{|c|c|}
\hline COMPONENTES & INDICADORES \\
\hline \multirow{3}{*}{$\begin{array}{l}\text { APRENDIZAJES DE } \\
\text { CALIDAD }\end{array}$} & $\begin{array}{l}\text { DOCENTES MOTIVADORES que despiertan el } \\
\text { entusiasmo de los estudiantes por aprender. }\end{array}$ \\
\hline & $\begin{array}{l}\text { CHICOS Y CHICAS TIENEN LAS MISMAS } \\
\text { OPORTUNIDADES DE APRENDER, ambos reciben un } \\
\text { trato justo, sin estereotipos de género, favoritismos ni } \\
\text { discriminación. }\end{array}$ \\
\hline & $\begin{array}{l}\text { MAESTRAS y MAESTROS que atienden en relación a } \\
\text { la diversidad del aula, brindando aprendizajes según } \\
\text { sus necesidades especificas de sus alumnos. }\end{array}$ \\
\hline PARTICIPACIÓN & $\begin{array}{l}\text { PADRES Y MADRES COMPROMETIDOS EN LA VIDA } \\
\text { DE LA ESCUELA que apoyan el aprendizaje de sus } \\
\text { hijos e hijas, participan en las reuniones de aula y de } \\
\text { Escuela para Padres y Madres. }\end{array}$ \\
\hline $\begin{array}{l}\text { SALUD, NUTRICIÓN } \\
\text { BUEN TRATO }\end{array}$ & $\begin{array}{l}\text { SEGURO DE SALUD, todos los estudiantes cuentan } \\
\text { con Seguro Integral de Salud u otro similar. }\end{array}$ \\
\hline
\end{tabular}

Una vez priorizados los indicadores se organizan los grupos para diseñar y aplicar los instrumentos, esta selección de instrumentos va a depender de las características de la escuela; se pueden aplicar encuestas, foto videos, video evaluación, grupo focal, escalas de valoración, campo de fuerza, análisis documental, etc.

En este paso, es fundamental que los responsables de aplicar seleccionen un instrumento que conozcan, y manejen el procedimiento en su aplicación, por eso hay libertad en la selección; sin embargo, se debe tener en cuenta prioritariamente en su construcción o selección el "qué queremos evaluar" y "a quién está dirigido".
Paso3-Elaboración de los planes de mejora

Después de la valoración de los resultados corresponde la toma de decisiones respecto a la definición de los problemas que serán atendidos en forma prioritaria y proponer las acciones de mejora.

En esta etapa participan de forma directa todo el personal de la institución educativa liderado por el CONEI. Son ellos los que, con la asistencia del acompañante externo, deberán identificar los problemas prioritarios. Una vez identificados los problemas prioritarios se deben plantear las acciones para su mejora, las mismas que serán incorporadas a los planes de trabajo del centro. Se deberá elegir responsables directos de conducir la realización de dichas acciones. Asimismo, las acciones deben ser comunicadas a las personas implicadas en su ejecución.

\section{Paso 4-Monitoreo}

Este proceso de implementación de los planes de mejora debe ser acompañado a través de un monitoreo del equipo responsable de la ejecución de los planes de mejora, a fin de ir conociendo la efectividad de la propuesta y los diversos obstáculos que se presentan en su implementación.

Para Stenhouse (1984), “...lo que mejora la acción educativa no es tanto la investigación sobre los docentes, sino la de los docentes al analizar su actividad", podemos ver entonces que este es un proceso donde los integrantes de la institución educativa de manera participativa y organizada coordinan acciones, a partir de indicadores amigables de calidad educativa, que les permite sentar las bases del mejoramiento de la calidad del servicio educativo que brindan.

Según los datos brindados por la Unidad de Gestión Educativa Local de Ventanilla, las escuelas EFI de la red norte de Villa de los Reyes, son las escuelas con mayor participación y experiencia en procesos de autoevaluación institucional, las escuelas del nivel primaria han elevado sus logros de aprendizaje, según esta data el nivel de logro de aprendizaje de los estudiantes de la red ha crecido durante el periodo 2012-2015 
en un $65 \%$ en comprensión lectora y en matemáticas en un 45\%; también, las escuelas del nivel inicial han implementado una propuesta pedagógica acorde a las características y necesidades evolutivas de los estudiantes, enfatizando en la psicomotricidad. En la red se encuentran 12 defensorías escolares, se promueven estilos de vida saludable, el cumplimiento del derecho a la identidad de los estudiantes y han desarrollado capacidades para lagestión de riesgos.

A manera de conclusión, podemos decir que cada escuela perteneciente al modelo educativo de "Escuelas Felices e Integrales", de la red norte de Ventanilla, es única, y diferente a cualquier otra y como grupo es una organización singular ya que la escuela está formada por un colectivo de personas en el que confluyen diversos intereses, por ello, tendríamos también que tener en cuenta en relación a la implementación del proceso de autoevaluación, la iniciativa, interés y participación de los actores educativos, permanencia y estilos de gestión, e institucionalización de los procesos de autoevaluación en cada escuela, ya que el proceso de autoevaluación planteado en el modelo de Escuelas Felices e Integrales es una experiencia previa muy importante que las instituciones educativas pueden articular con los procesos planteados por el Instituto Peruano de Evaluación, Acreditación y Certificación de Educación Básica (IPEBA) e iniciar el camino hacia la acreditación.

El propósito de hacer evidente el nivel de avance en los procesos de autoevaluación escolar y la participación de los actores educativos con la finalidad que los resultados pudieran utilizarse dentro de la región sin pretender ser modelo, sino simplemente una guía para aplicar la autoevaluación en otras instituciones educativas.

\section{REFERENCIAS}

Delgado, E. (2013). Construyendo Escuelas Felices e Integrales. Municipalidad de Ventanilla.

IPEBA (2010). Propuesta del uso de la acreditación como herramienta que cierre brechas de inequidad en el acceso a la educación de calidad. Perú: Ministerio de Educación - Dirección General de Educación Intercultural Bilingüe y Rural. http://www.unicef.org /spanish/publications/index_49574.html
IPEBA/Instituto Peruano de Evaluación, Acreditación y Certificación de la Calidad de la Educación Básica (2012). Matriz de evaluación para la acreditación de la calidad de la gestión educativa de instituciones de educación básicaregular.Lima.

Neyra, C. (2010). Guía de Elaboración del Proyecto Educativo Institucional articulado al Proyecto Educativo Local de Ventanilla. Lima: Municipalidad Distrital de Ventanilla. 